\title{
The Dynamics of National Specialization
}

\author{
Giovanni Peri; Universita’ Bocconi and IGIER \\ first draft — October 22, 1999 \\ this draft - January 16,2000
}

\begin{abstract}
I explore the dynamics of national production in a two-sector, two-country model with costly cross-sector mobility and forward-looking agents, when trade costs fall. If economic integration promotes potential gains from specialization, it is the actual decision of workers to switch sector and the efficiency of the reallocation process to determine the dynamics of specialization. Using the phase diagram method, introduced by Baldwin $1999 \mathrm{I}$ am able to consider the optimal choice of the representative agent, which will determine the dynamics of national specialization. I discover some important and interesting features of the equilibria and of their stability properties, which would have been completely overlooked by the "simple" economic geography models. In particular I find that, lacking comparative advantage, specialization may not take place at all when trade costs decrease, if the costs of moving labor across sectors are too high.
\end{abstract}

JEL Classification Codes: R1, R3, F16

Address of the author:

Giovanni Peri, Dipartimento di Economia, Universita' Bocconi and IGIER 20136 Milan, Italy. E-mail: giovanni.peri@uni-bocconi.it

${ }^{*}$ I thank Luisa Lambertini for very useful and insightful conversation leading to our joint work and to this paper. 


\section{Introduction}

The process of emergence of specialization and trade patterns in a world in which trade costs are decreasing and "free-ness" of trade is increasing, has attracted vast attention and research in the modern trade and geography literature both from a theoretical and an empircal point of view. The empiricists are interested in the consequences of decreased trade costs on the patterns of national production and trade (for example to understand European evolution after the EMU ${ }^{1}$ ). The theorists are eager to sharpen the tools of the "new economic geography" models, making them fully dynamics and introducing fully optimizing forward-looking agents. This would open the horizon to rigorous formal analysis of extremely important phenomena such as labor migration across regions, booms and collapses of industries, time evolution of international specialization. The first and pioneering models in the "new geography" literature, in fact ${ }^{2}$, have shown us comparative static analysis, filling with some educated guess the dynamics in between. The new models (such as Baldwin [3]) should develop fully fledged dynamic analysis, and enable us to study rigorously the adjustment process and the transition between equilibria, which will be extremely important, especially as the process may take some time. This paper is a contribution in this sense.

The rigorous dynamic analysis of the emergence of specialization patterns in a country allows to define the stability properties of the equilibria, the evolution and speed of adjustment towards them and their welfare characteristics. Moreover some concepts such as the cost of migration, or the cost of the search process in finding a new job could be made clear in this context and their consequences studied. The results obtained with the fully dynamic model studied in this paper are interesting and striking. Our analysis focuses on the case of industrial specialization in a twocountry, two-sector model. We allow three forces to operate in such a model, and their interactions generate the dynamics of specialization as trade costs decrease.

First, the two sectors have backward and forward linkages as the production of each variety in a sector of production uses other varieties as intermediates and these forces generate agglomeration economies. Second, there are frictional costs of moving factors of production from one sector to the other which we can imagine as determined by labor market rigidities or by cost of re-qualifying for another sector. Third, in the second part of the paper, we introduce comparative advantages as a further force driving international specialization, as the two sectors could be intensive in different factors and the two countries are differently endowed.

In this paper we develop the analysis of the effects of "globalization" , on the spe-

\footnotetext{
${ }^{1}$ For a review of the empirical litearture on Europe and for references see Aigigner [1] and WolfmayrSchnitzer [11]

${ }^{2}$ Namely the models by Krugman [8], [9] Krugman and Venables[10], now summarized in Fujita et al. $[6]$
} 
cialization of a country, assuming that changing jobs is costly, that households are forward looking and optimizing and move their labor supply between sectors (but not across countries) in order to maximize their lifetime utility. In the considered framework I will analyze the effect of an unanticipated drop in trade cost that generates a boom in a sector, and the effect of an anticipated one. I will also consider how the specialization process changes when there are two types of labor (skilled and unskilled) which are used with different intensity in the two sectors. The only exogenous force in our model is the globalization process, namely the fact that "free-ness" of trade across countries increases due to lower barriers to trade. All the other events described, such as the boom of a sector, its reversal, the dynamic evolution of specialization are all endogenously generated by the model. This allows a very complete and thorough analysis and also enable us to think of the chain of dynamics processes as all endogenously generated.

The key insight of this model can be easily rationalized. It is the interaction of the above-mentioned forces, comparative advantages, agglomeration economies and labor market frictions, which determines the dynamics of specialization. When trade cost are too high, the agglomeration economies are not strong enough and the comparative advantage of countries generate incomplete specialization, as in the Hecksher-Ohlin model with trade costs. This is a stage of low trade "free-ness" and relatively despecialized national economies. Then, when trade costs decrease and agglomeration economies pick momentum, the intensity of frictional cost on the labor market and the intensity of comparative advantages become key to determine the evolution of a country's specialization pattern. On one hand, if there are large costs of moving labor across sectors (due, say, to high search costs and labor market rigidities) and low comparative advantage forces, then the economies may remain non-specialized, without generating welfare improving agglomeration economies. On the other hand, if frictions are not too high, or comparative advantages are significant, the economies will fully specialize and enjoy higher welfare as transport cost decrease. We also show that in this last case the specialization process may begin before the actual drop in trade cost, as the result of common expectation anticipating such drop.

The paper is organized as follows: Section 2 briefly describes the static model with one type of labor and analyzes the dynamics of this model when agents are forward looking. Section 3 presents the model with skilled and unskilled workers and comparative advantages in the two countries and Section 4 analyzes its dynamics when the agents are forward looking households. Section 5 concludes the paper. 


\section{The Model with one type of Labor}

\subsection{The Static Model}

We consider two countries, each with two industries, and only one factor of production, say labor, along the lines of the model in Fujita et al. [6], Chapter 16. As the static model is standard, it will be laid out very briefly in the present section for the Home economy; the Foreign economy is completely symmetric and the tilde character on top of a variable will denote that it is relative to the foreign economy. Each country is endowed with one unit of labor, which we assume is inelastically supplied by workers. Labor may be employed in either of the two industries, which we label industry 1 and 2 , but it cannot move internationally.

Both industry 1 and 2 produce manufacturing goods and are monopolistically competitive. Each variety in either industry has a Cobb-Douglas technology that utilizes labor and intermediate goods both from their own industry and from the other industry, but crucially, a variety uses more intermediates from the own industry rather than from the other. On the demand side, all consumers are identical with demand elasticity for each variety in either industry equal to $\sigma$.

Each worker/consumer maximizes a utility function of the following type:

$$
U=X_{1}^{0.5} X_{2}^{0.5}
$$

where $X_{1}$ is a composite index of the consumption of varieties (goods) manufactured by industry 1 and $X_{2}$ is a composite index of the consumption of goods manufactured by industry 2 .Defining with $x_{i}(j)$ the consumption of each variety $j$ produced in industry $i$. The consumption index $X_{i}$ is defined by:

$$
X_{i}=\left[\int_{0}^{n_{i}} x_{i}(j)^{\frac{\sigma-1}{\sigma}} d j\right]^{\frac{\sigma}{\sigma-1}}, \quad \sigma>1
$$

It is easy to recognize that (2) is a constant-elasticity-of-substitution function where $\sigma$ is the elasticity of substitution between any two varieties. $n_{i}$ is the number of varieties produced in industry $i$.

The price index for the composite consumption good produced in industry $i$ (i.e. corresponding to (2)) is denoted by $G_{i}, i=1,2$ and it is equal to

$$
G_{i} \equiv\left[\int_{0}^{n_{i}} p_{i}(j)^{1-\sigma} d j\right]^{\frac{1}{1-\sigma}},
$$

where $p_{i}(j)$ is the price of each manufactured good $j$ produced in industry $i=1,2$. Notice that $G_{i}$ measures the minimum cost of purchasing a unit of the composite good 
$X_{i}$. The compensated demand function for the $z$-th variety produced in industry $i$ is, therefore:

$$
x_{i}(z)=x_{i}=\left[\frac{p_{i}(z)}{G_{i}}\right]^{-\sigma} X_{i} .
$$

where, because of the symmetric spending of consumers' income $(Y)$ in the two sectors, we have:

$$
X_{i}=\frac{Y}{2 G_{i}}, \quad i=1,2 .
$$

Shipping goods between the two countries involves iceberg "trade" cost $T>1$ in the sense that only a fraction $1 / T$ of the shipped good arrives to destination ${ }^{3}$. The production function for the variety $j$ in industry $i$ require a fixed amount $F$ (overhead fixed costs) and variable inputs according to the production function:

$$
c_{i}^{m}(j) x_{i}(j)+F=l_{i}(j)^{\beta} X_{i}^{\alpha} X_{-i}^{\gamma}, \quad i=1,2,
$$

where $\alpha+\gamma+\beta=1, l_{i}(j)$ is labor employed for the production of variety $j$ and $-i$ indicates the sector but $i$. We are going to assume that $\alpha>\gamma$, which implies that the links within the same industry are stronger than the links between them. This is the assumption that makes co-location of firms in the same sector desirable. We can think of the industry linkages as "strictly speaking" input-output linkages, but they could also involve knowledge flows, if knowledge and information is passed on with the trade of intermediates.

Given this production function and the monopolistic competition structure of the market the optimal pricing is:

$$
p_{i}(j)=w_{i}^{\beta} G_{i}^{\alpha} G_{-i}^{\gamma}
$$

where we have chosen units so that the marginal input requirement equals the pricecost mark-up $\left(c^{m}=(\sigma-1) / \sigma\right)$ and we have denoted with $w_{i}$ the wage rate paid in industry $i$. Equation (7) shows that firms set prices (net of taxes) as a fixed mark-up over the marginal cost of production (for our choice of parameters the mark-up is zero). We suppose there is free entry and exit in response to profits; hence, the zero-profit condition implies that the equilibrium output for firm $j$ in sector $i$ is

$$
x^{*}=F \sigma .
$$

By choosing the fixed cost appropriately so that $x^{*}=1 / \beta$, the total labor income in industry $i$ is given by

$$
w_{i} l_{i}=n_{i} p_{i}
$$

\footnotetext{
${ }^{3}$ We define this generally as "trade cost", they may be literally transport cost or trade barriers that involve some cost of trading goods between countries.
} 
where $l_{i}$ is the labor share employed in industry $i$ and $l_{i}+l_{-i}=1$, consistent with our assumption that the Home labor force is unity. Using (3) we can write the price index for the Home economy in industry $i$ as

$$
G_{i}^{1-\sigma}=l_{i} w_{i}^{1-\beta \sigma} G_{i}^{-\alpha \sigma} G_{-i}^{-\gamma \sigma}+\tilde{l}_{i} \tilde{w}_{i}^{1-\beta \sigma} \tilde{G}_{i}^{-\alpha \sigma} \tilde{G}_{-i}^{-\gamma \sigma} T^{1-\sigma},
$$

which holds for $i=1,2$. An analogous expression holds for the price index of industry $i$ in the Foreign economy. Total expenditure from the Home country in industry 1 and two are:

$$
\begin{aligned}
& E_{1}=\left[\frac{w_{1} l_{1}+w_{2} l_{2}}{2}\right]+\left[\frac{\alpha w_{1} l_{1}+\gamma w_{2} l_{2}}{\beta}\right], \\
& E_{2}=\left[\frac{w_{2} l_{2}+w_{1} l_{1}}{2}\right]+\left[\frac{\alpha w_{2} l_{2}+\gamma w_{1} l_{1}}{\beta}\right] .
\end{aligned}
$$

At last, the market-clearing condition for industry $i$ in the Home economy is

$$
\left(w_{i}^{\beta} G_{i}^{\alpha} G_{-i}^{\gamma}\right)^{\sigma}=\beta\left[E_{i} G_{i}^{\sigma-1}+\tilde{E}_{i}\left(\tilde{G}_{i}\right)^{\sigma-1} T^{1-\sigma}\right] .
$$

By the Walras' law, the market clears for industry $-i$ in the Home country; a marketclearing condition similar to (13) holds for the Foreign economy.

The equilibrium conditions consist of nine equations for the Home country and, of course, nine equations for the Foreign country. The nine equations for the Home economy are: the market-clearing condition (13), one for each industry; the equilibrium price index (10), one for each industry; the spending equations (11) and (12) and a price normalization, which we have chosen as the wage in industry 1 , i.e. $w_{1} \equiv 1$. These nine equations determine nine endogenous variables: $w_{2}, G_{1}, G_{2}, p_{1}, p_{2}, g_{1}, g_{2}, E_{1}$ and $E_{2}$. The symmetry of the set-up guarantees that the variables in foreign, relative to sector 1 are identical to the variables in home relative to sector 2 and vice-versa.

The solution to this system gives the wage in each sector (at Home and in Foreign) as a function of $l_{1}$ (and $\tilde{l_{1}}$ ). We have that the real wage in sector $i$ is also the instant indirect utility for a worker and is given by:

$$
\omega_{i}=\frac{w_{i}}{G_{1}^{0.5} G_{2}^{0.5}}
$$

We can now simulate the behavior of the variables $\left(\omega_{1}-\omega_{2}\right)^{4}$ which is the difference in real wages for the home country and plays a key role in the determination of the dynamics of the model. In particular, this function gives, for each level of $l_{1}$, the wage differential between the two sectors, which represents the incentive to move from one

\footnotetext{
'The parameters' value of the simulation are given in the Appendix
} 
sector to the other. I plot for the parameters' value reported in the appendix, the function $\left(\omega_{1}-\omega_{2}\right)$, which will be later called $\Omega\left(s_{L}\right)$, as a function of the share of labor in sector one in the home economy $\left(s_{L}\right)$. For high value of the trade cost parameter $(\mathrm{T}=1.9)$ the function is as in Figure 1 with only the symmetric point with equal wages in the two sectors.

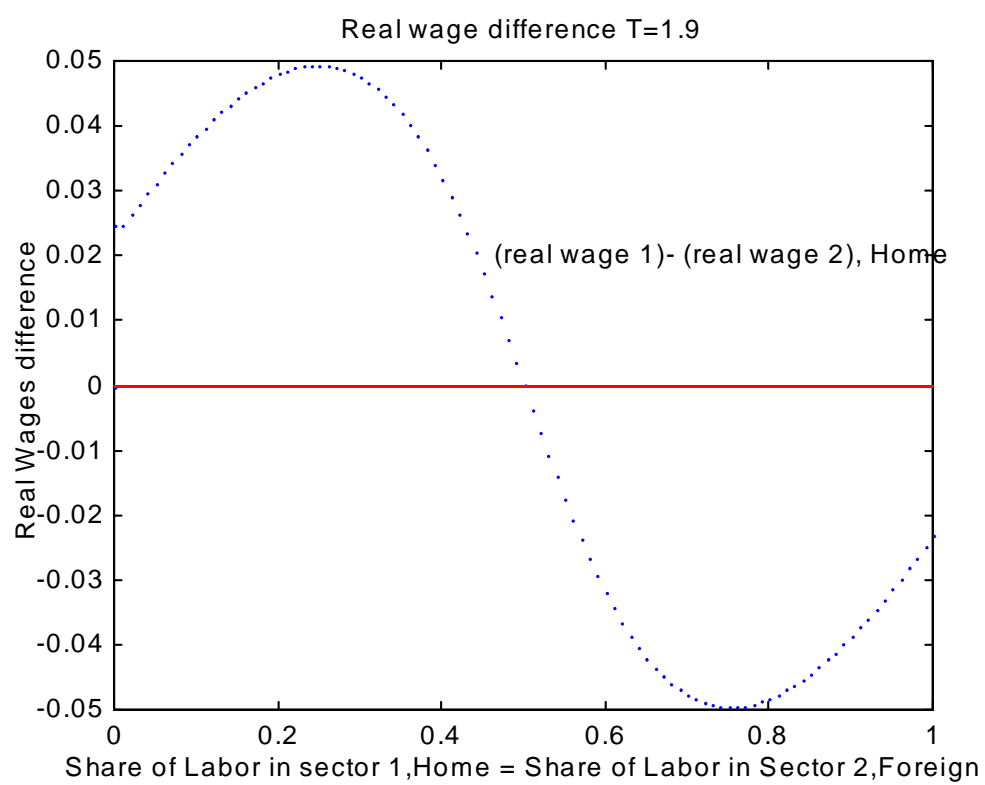

Figure 1: $\Omega\left(s_{L}\right)$ for $\mathrm{T}=1.9$

For lower trade cost ( $\mathrm{T}=1.7$, see Figure 2 below) there will be three values for which $\Omega\left(s_{L}\right)=0$ and in the fully specialized cases the sector attracting the whole production will also be the one paying higher real wages $(\Omega(0)<0, \Omega(1)>0)$.

Finally for even lower trade cost, $(\mathrm{T}=1.5)$ the function $\Omega\left(s_{L}\right)$ will be upward sloping, still equal zero in the symmetric equilibrium, and still with the sector attracting all production paying higher wages in the fully specialized case. This situation is illustrated in Figure 3 below.

\subsection{Equilibria, Dynamics and Trajectories of Specialization}

Having described the static model we are able to describe the equilibrium with infinitelylived forward looking households. We assume many identical households, who are endowed with one unit of labor and may offer labor in either sector or part in one sector 


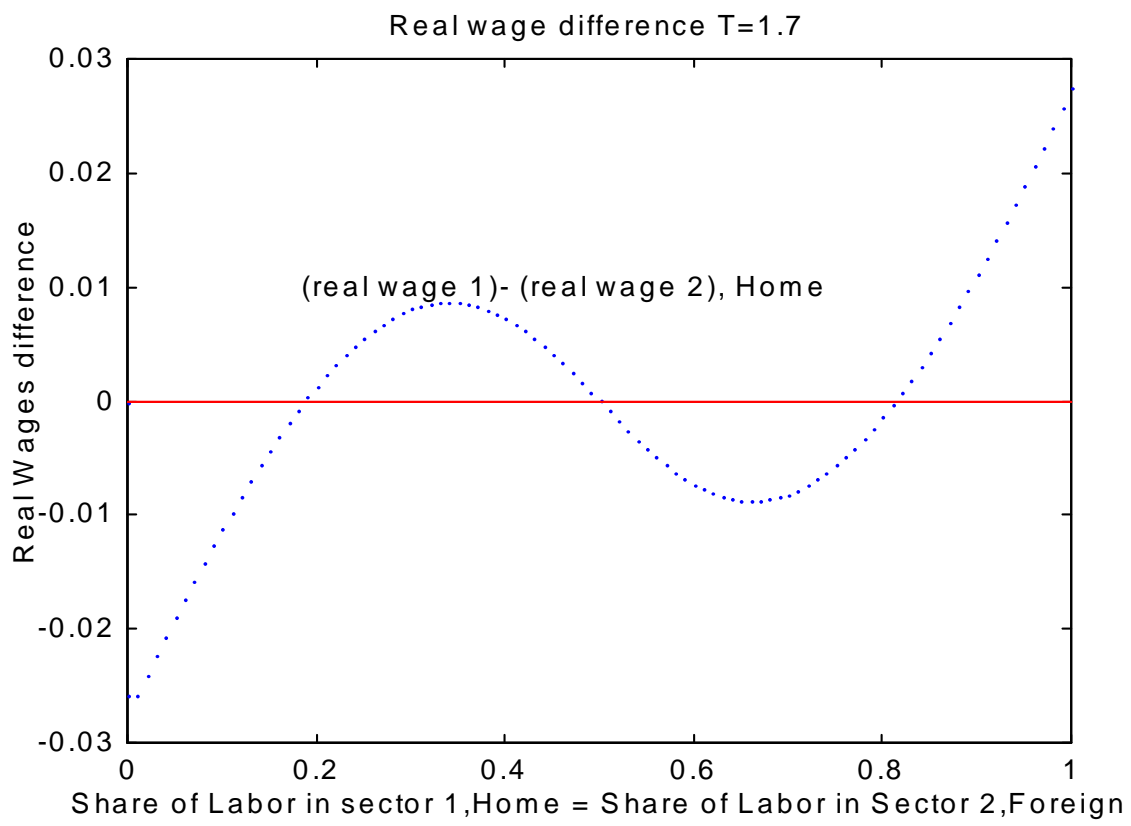

Figure 2: $\Omega\left(s_{L}\right)$ for $\mathrm{T}=1.7$

and part in the other. In shifting hours of work from one sector to another these households incur in a cost due to, say, a search process in the industry to which they are moving their labor or to an adjustment cost in the new sector. This cost is reasonably increasing with the speed at which they are moving their labor supply from one sector to the other. The justification for this assumption is that, in order to transfer labor faster the household need to employ more costly resources in the search or in the re-training process.

The instant utility of a worker is given by the instant real wage less a quadratic cost of "changing sector". Assuming an inter-temporal discount rate equal to $r$ we may write the lifetime utility of the $i-t h$ household as:

$$
\int_{t=\boldsymbol{o}}^{\infty} e^{r t}\left(l_{1 i} \omega_{1}+\left(1-l_{1 i}\right) \omega_{2}-\frac{\gamma m^{2}}{2}\right) d t
$$

where $\omega_{1}$ and $\omega_{2}$ are the real salaries in industry one and two, $l_{1 i}$ and $\left(1-l_{1 i}\right)$ are the amount of labor supplied in each industry by the $i$ - th household, $r$ is the discount and interest rate and $\gamma$ is a parameter capturing the cost of adjustment to the other sector and $m$ is the instant rate at which the labor supply is moved from industry two to industry one. In the aggregate, as both the single household's and the whole economy's 


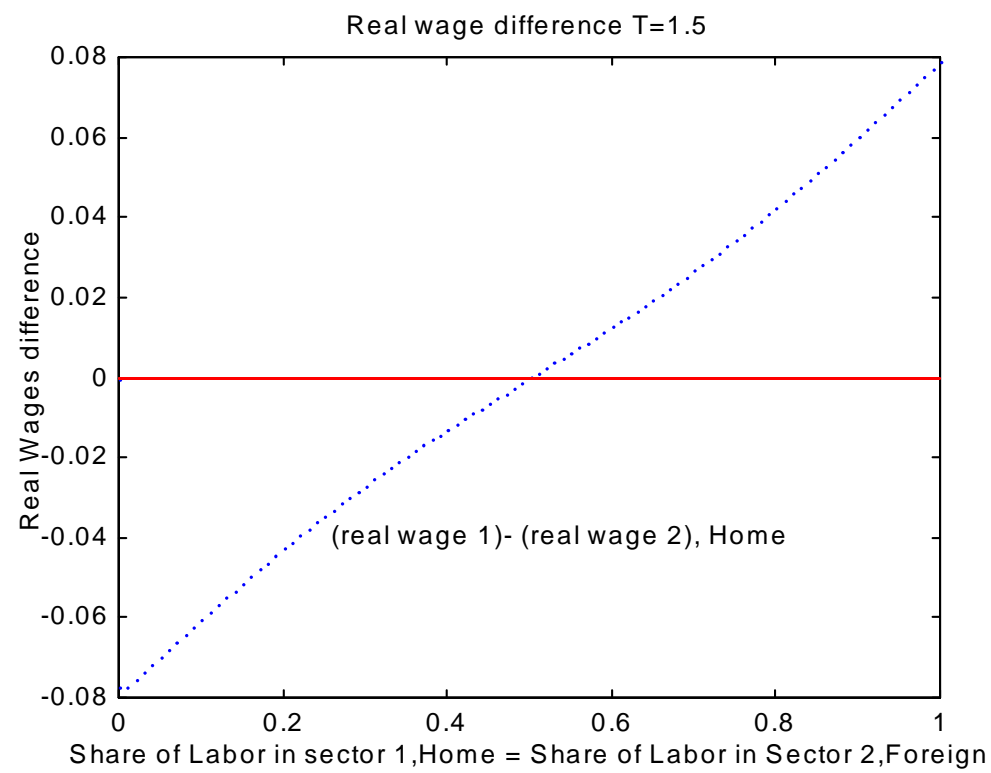

Figure 3: $\Omega\left(s_{L}\right)$ for $\mathrm{T}=1.5$

labor supply have measure one, $\dot{L}_{1}=m$. The parameter $\gamma$ captures the rigidities of the local labor market, as moving from one sector to another will generate more disruption and frictions in the short run, the less efficient and flexible the market is. Shifting labor rapidly is very costly if the labor market has frictions, rigidities and lags. The optimal programming problem, solved by a household, implies the maximization of the following current-value Hamiltonian:

$$
H=l_{1 i} \omega_{1}+\left(1-l_{1 i}\right) \omega_{2}-\frac{\gamma m^{2}}{2}-\lambda m
$$

The first order and transversality conditions for optimality are:

$$
\begin{gathered}
\gamma m=\lambda \\
\dot{\lambda}=r \lambda-\left(\omega_{1}-\omega_{2}\right) \\
\lim _{t \rightarrow \infty} m \lambda e^{-r t}=0
\end{gathered}
$$


Manipulating only slightly these conditions, and calling the share of labor in sector one with $\left(s_{L}\right)$, we have the two equations that define the dynamics of the aggregate system:

$$
\begin{gathered}
\dot{s_{L}}=\frac{\lambda}{\gamma} \\
\dot{\lambda}=r \lambda-\left(\omega_{1}-\omega_{2}\right)
\end{gathered}
$$

with the restriction $0 \leq s_{L} \leq 1$. So that whenever we reach the boundaries, then the feasible rate of change of $s_{L}$ will be 0 , if $s_{L}$ would be increasing at the point $s_{L}=1$ or if it would be decreasing at the point $s_{L}=0$.

If we linearize this system, defining $\left(\omega_{1}-\omega_{2}\right)=\Omega\left(s_{L}\right)$, we will have the following system of differential equations:

$$
\left[\begin{array}{c}
\dot{s_{L}} \\
\dot{\lambda}
\end{array}\right]=\left[\begin{array}{cc}
0 & \frac{1}{\gamma} \\
-\frac{d \Omega}{d s_{L}} & \rho
\end{array}\right]\left[\begin{array}{c}
s_{L} \\
\lambda
\end{array}\right]
$$

The local stability of the equilibria can be analyzed, by finding its characteristic roots, and characteristic vectors for this linearized system, around those equilibria. Nevertheless the important analysis has to take into account global stability properties, given that the relevant changes in this model happen, when the equilibrium passes from the de-specialized to the fully specialized one. We will use a combination of the Phase diagram and of the eigenvalues techniques to assess the stability characteristics of the equilibria. Let me first consider the phase diagram for this problem and then I will define the optimal transition from the de-specialized to the specialized equilibrium adopting the above framework. To avoid the complication of the existence of intermediate unstable equilibria, between the de-specialized and the specialized equilibrium we assume that the parameter capturing trade costs will drop from a value which sustains the de-specialized equilibrium (as in Figure 1) to one which sustains the specialized one (as in Figure 3), as the consequence of one only change and not progressively. This assumption can be rationalized as trade cost may change in a 'discrete' way as consequence of international agreement (for example the 1992 Maastricht treaty in Europe removed all the barriers to trade of goods).

\subsubsection{Phase Diagram in the de-specialized equilibrium}

Let's consider the de-specialized equilibrium first. Its phase diagram is reported in Figure 4 below. 


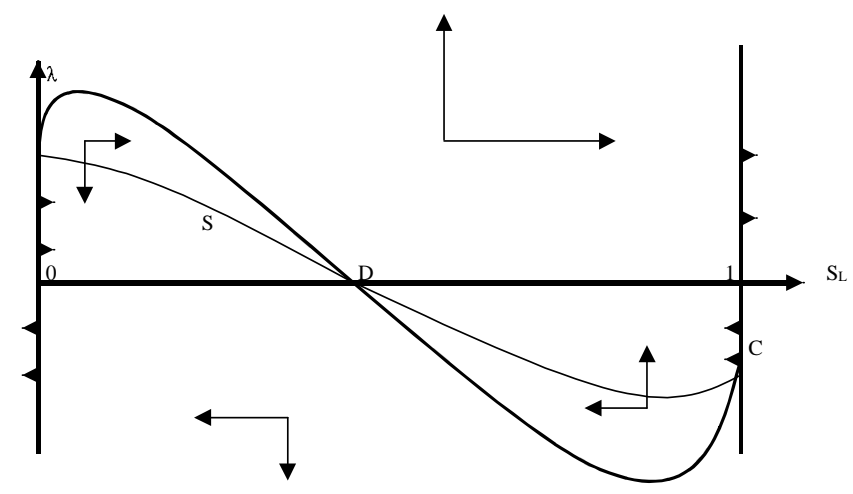

Figure 4: Phase diagram for high transport cost

The de-specialized, symmetric equilibrium, D, is saddlepath stable, and this can be formally shown considering the characteristic roots of the system 22 , which are as follows:

$$
\mu_{1,2}=\frac{r \pm \sqrt{r^{2}-\frac{4}{\gamma} \frac{d \Omega}{d s_{L}}}}{2}
$$

As $\Omega\left(s_{L}\right)$, has a negative first derivative in the symmetric equilibrium, this ensures the existence of one positive and one negative characteristic root, which characterize a saddlepath stable equilibrium. Under our assumption no other steady state equilibrium exists. If $s_{L}=1$ or $s_{L}=0$, any choice of $\lambda$, except for the one on the saddlepath $\mathrm{S}$, generates unstable trajectories which violate the transversality condition. Therefore, if for some reason the system begins in a point as $\mathrm{C}$, it will evolve towards the symmetric equilibrium.

\subsubsection{Phase Diagram when Full Specialization is Feasible}

Let's now assume that the system unexpectedly experiences a decrease in trade cost say from a level around $\mathrm{T}=1.9$ to a level $\mathrm{T}=1.5$, jumping for simplicity the intermediate case, and transforming the function $\Omega\left(s_{L}\right)$ as in Figure 3, making the agglomeration state potentially sustainable. What will happen?

The outcome of this change will crucially depend on the cost of moving factors across sectors, i.e. on the degree of flexibility of the market. We assume that the system is in the symmetric equilibrium when the drop in trade cost takes place. We need, therefore, to study the stability properties of this equilibrium, now that the function $\Omega\left(s_{L}\right)$ has 


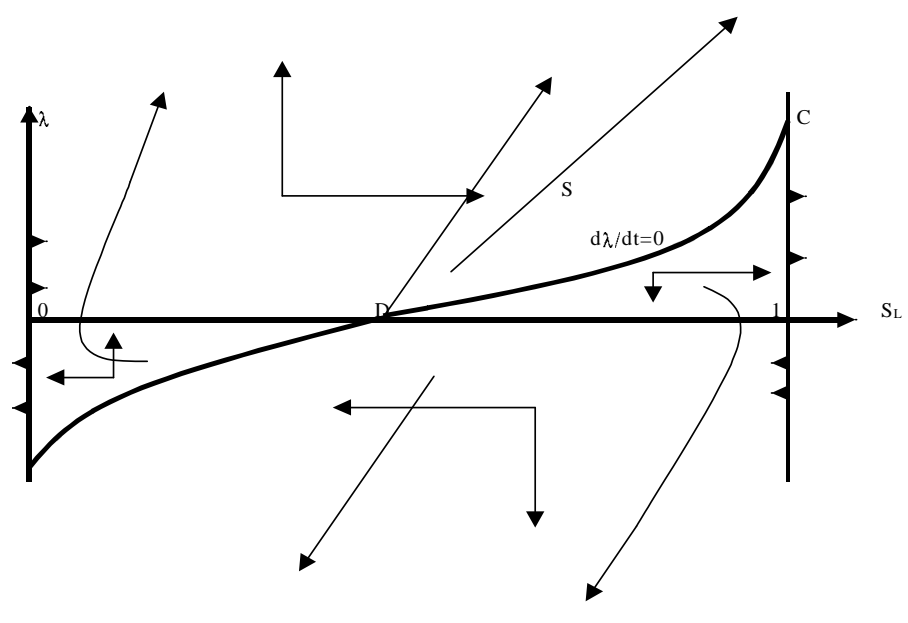

Figure 5: Low transport cost, high labor market rigidities

changed. The characteristic roots, defined by 23 can be of two types. If $0<\frac{d \Omega}{d s_{L}}<\frac{\gamma r}{4}$, i.e. when the cost of switching sector are high compared to the change in value of $\Omega$, then we will have that both characteristic roots are positive and real. This implies that the solution trajectories will be always increasing in both $\lambda(t)$ and $s_{L}(t)$. This rules out the possibility that one trajectory will cross the line $d \lambda / d t=0$ as that would imply a decrease in $\lambda$ after that. The dynamics will therefore be as in Figure 5 in this case, with only "exploding paths". If we define as equilibrium a situation, which satisfies the first order conditions, and in which the variables asymptotically converge to constant values, in this case the only equilibrium is given by the situation in which the system stays on the symmetric equilibrium, D, as any other trajectory is unstable and would be sustained only by ever decreasing or ever increasing expectations.

This situation, therefore, introduces a very novel feature in this type of model, not understandable in the static version. If the cost of moving labor across sectors is high, then the trajectories consistent with the first order conditions, such as trajectory $\mathrm{S}$ in Figure 5, imply an increase in the "shadow value" of changing sector $(\lambda)$, too large, on the adjustment path. This value will be incompatible with the level actually attained, once full agglomeration is reached, in a point like C. Therefore no "rational" trajectory which satisfies the first order conditions for optimality will lead to agglomeration, into $\mathrm{C}$, and therefore the only "saddlepath stable" optimal equilibrium is to remain in D. Such result is very striking: the symmetric de-specialized equilibrium, which was defined, although not rigorously, as locally unstable in the static analysis a'-la Fujita 
el al $[6]$, is now the unique saddlepath stable equilibrium.

If cost of moving across sectors, are not so high, namely if $\frac{d \Omega}{d s_{L}}>\frac{\gamma r}{4}$, the symmetric equilibrium will have two complex roots, whose real part is certainly positive and therefore the solutions will be cyclical but spiraling out of this equilibrium. In particular if they satisfy the above inequality but they are not too low (we will call this case of intermediate cost of changing sector, and we have represented this in Figure6 ) we will have a trajectory, out of the symmetric equilibrium which gradually approaches the fully agglomerated point $\mathrm{C}$. This is the only trajectory, which satisfies the first order conditions, and brings to a new steady state with fulfilled expectation on the "shadow value of changing sector".

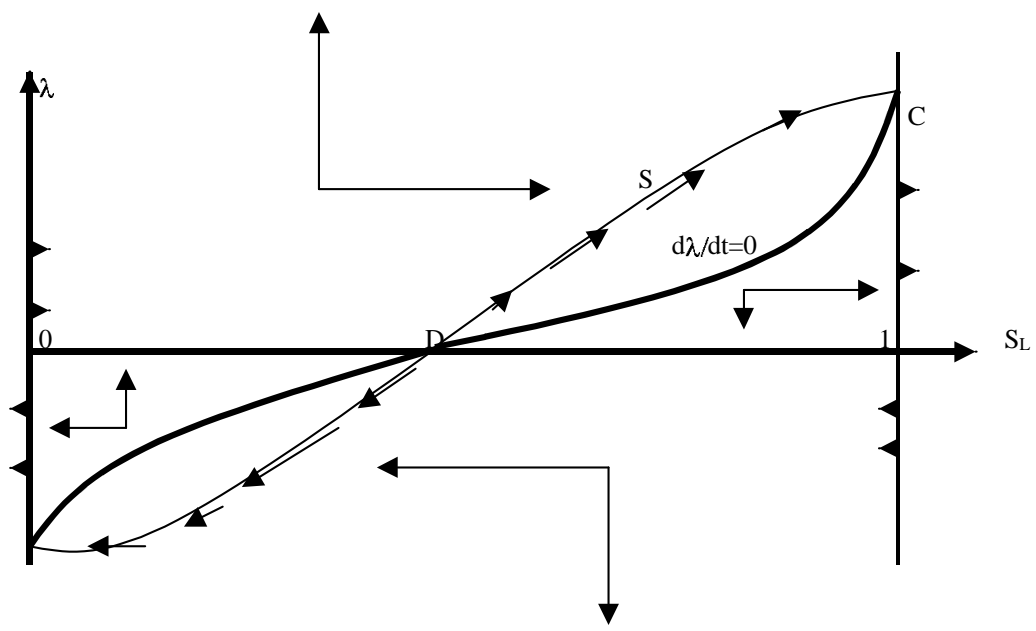

Figure 6: Low transport cost, Intermediate labor market flexibility

Finally if the cost of changing sector are particularly low, we may have a situation described in Figure 7. In this case the only path that leads to $\mathrm{C}$ is spiraling already in the interval between the point D and C. Therefore the optimal choice of the agent, as soon as the lower trade cost are introduced, is to jump to the point $\mathrm{B}$, anticipating higher value of migration, and rapidly move toward $\mathrm{C}$, fulfilling the expectations of gains from migrations. This equilibrium, which is saddlepath stable, is the most reminiscent of the so called "catastrophic agglomeration" of the static models, as now the shadow value of migrating suddenly jumps up and people rapidly move to one sector. 


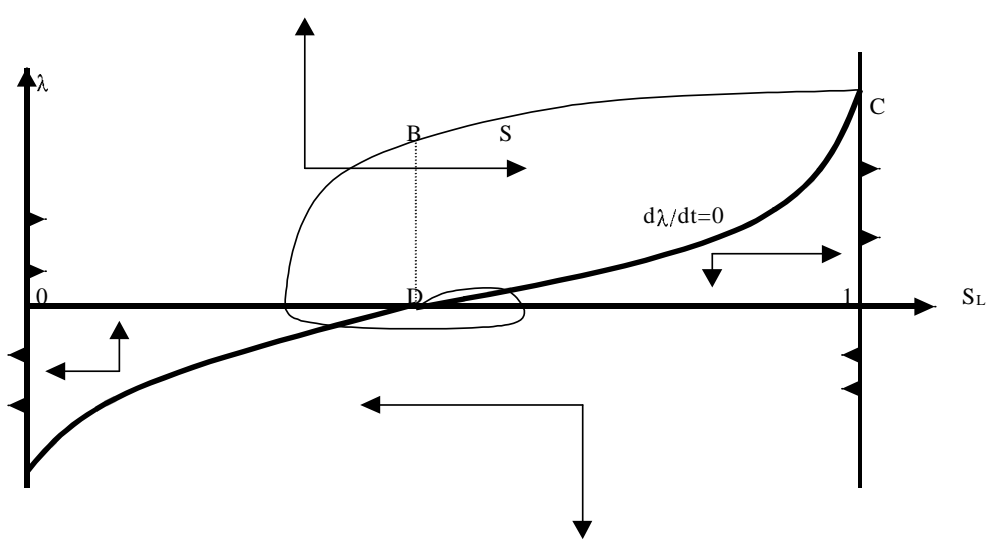

Figure 7: Low Transport Cost, Low Labor Market Rigidities

\subsubsection{Anticipated decrease of trade Cost}

With the framework developed in the previous section, we can now analyze the effects of anticipated changes. The model allows to deal rigorously with expectations, which will probably arise before an event takes place. We assume that, before the trade cost actually decreases, when the system is in the symmetric equilibrium, some expectations arise that sector 1 will be favored in the country once globalization takes place. In this case, even in the mere anticipation of the decrease in trade costs, workers should start to move into sector one, more or less rapidly, depending on the cost of changing sector, so that when the change will take place they are positioned on the path, leading to point $\mathrm{C}$. The trajectory, for the case of low rigidities in the labor market, is shown in Figure 8.

At $t_{0}$, the moment in which the expectation of future decrease in trade cost and boom in sector 1 is formed, the value of moving to sector one jumps up and workers start to move to this sector. The speed of moving and the subsequent expectations on the value of moving evolve in a way which ensures that at the time of the actual decrease in trade cost, $t_{1}$, the system is on the trajectory leading to $\mathrm{C}$, and will complete its specialization. In the case of intermediate costs of changing sector, illustrated in Figure 6, on the other hand, it is optimal to increase the shadow value of moving to the other sector, and start the move, only when the actual drop in trade cost takes place. All other trajectories, starting above the point $\mathrm{D}$, and moving according to the dynamics illustrated in Figure 6 between $t_{0}$ and $t_{1}$ cannot end up on the saddlepath $\mathrm{S}$, at time $t_{1}$.

This experiment is illuminating, for a case like Europe, whose process of integration 


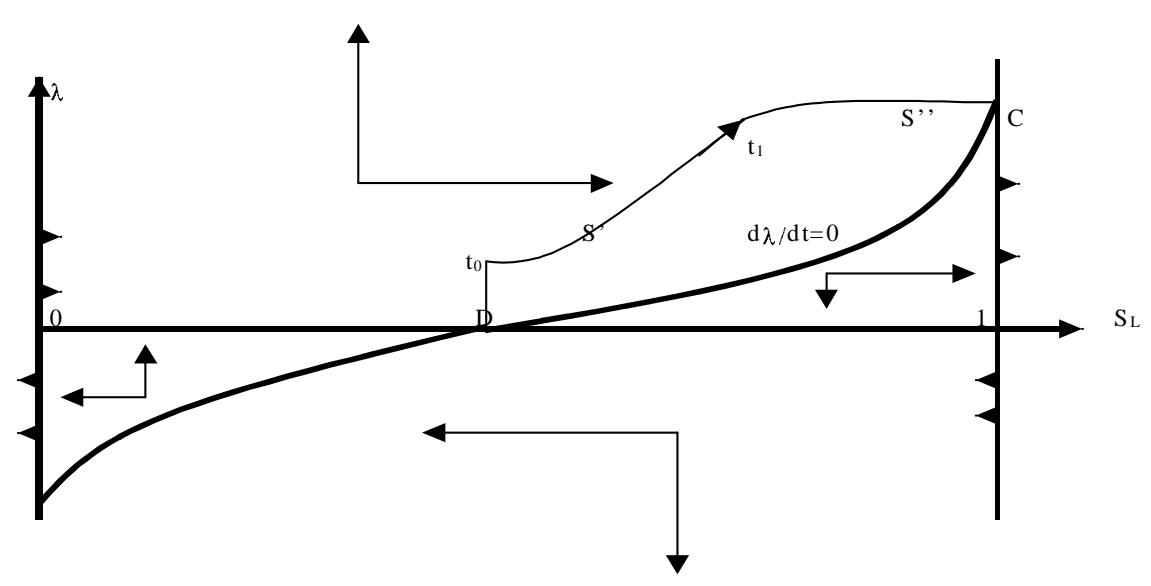

Figure 8: Adjustment to an anticipated drop in transport cost

could be anticipated, already 10 years ago. Under this circumstance a process of increased specialization should have taken place if the labor market institutions would have guaranteed the needed flexibility to decrease the cost of re-allocation. The fact that this tendency towards specialization does not arise until the mid eighties and at a rather slow pace (see Amiti[2]) could be another consequence of the lack of flexibility on the labor market in European countries. The present model, therefore offers a rational motivation for the delay in adjustment of forward looking agents, which may depend on the cost of changing sector.

\section{A Model with Skilled and Unskilled Workers and Comparative Advantages}

\subsection{The static Model}

The simple "static" model in the background of the previous section exhibits a "monotonic" behavior of agglomeration as a function of trade cost:s as these costs decreases the symmetric equilibrium becomes unstable and only full agglomeration is sustainable. In this section we want to allow for more complicated dynamics of agglomeration, which will add greater realism to the analysis. In particular there may be some "centrifugal" forces (such as congestion and price pressures) which, if trade costs decrease below a certain level, will promote the unraveling of the full agglomeration. One of these forces 
emerges if we have two, rather than one, factors of production and the two sectors are intensive in different factors. In this case, if the country endowment, is not too far from the world endowment, complete agglomeration may be putting too much pressure on the price of the factor used intensively. For trade cost falling below a treshold, a process of partial de-specialization may take place.

We consider in this section the two sectors-two factors (skilled and unskilled workers)two countries model. The relations defining the price indices, the price of each good, the expenditure in each sector and the market clearing conditions are exactly the same as equations $4,7,10,11,12$ and 13 in the previous section. The only difference is that the values $w_{1}, w_{2}$ which used to represent the wages in each sector, are now the total return to a labor-composite input, which is different in the two sectors. In particular the production function of sector $i$ is ${ }^{5}$ :

$$
c_{i}^{m}(j) x_{i}(j)+F=\left(\Phi l_{i}(j)^{\delta_{i} \beta} h_{i}(j)^{\left(1-\delta_{i}\right) \beta}\right) X_{i}^{\alpha} X_{-i}^{\gamma}, \quad i=1,2,
$$

where $\alpha+\gamma+\beta=1, \delta_{1}=\delta>0.5, \delta_{2}=(1-\delta)<0.5, l_{i}(j)$ and $h_{i}(j)$ are, respectively, unskilled and skilled labor employed for the production of variety $j$ and $-i$ indicates the sector other than $i$. $\Phi$ is a re-scaling constant. The production technology exhibits therefore different factor intensities: Sector 1 is more intensive in unskilled labor and sector 2 is more intensive (in a symmetric way) in skilled labor. An entrepreneur, by shifting skilled and unskilled labor from one sector to the other and selling the goods at the market price may get higher total return on total sales. Of course if this happens the production will be shifted to the more convenient production till the extra returns are eliminated. By choosing the constant $\Phi$ appropriately ${ }^{6}$, we can write a technological relation, between the cost of the labor composite used in sector $i, v_{i}$, and $r_{L}$ and $r_{H}$ the returns to skilled and unskilled labor, on the production possibility frontier, as follows:

$$
\begin{aligned}
& v_{1}=r_{L}^{\delta} r_{H}^{1-\delta} \\
& v_{2}=r_{L}^{1-\delta} r_{H}^{\delta}
\end{aligned}
$$

From the full employment condition, and the Cobb-Douglas function, we derive the two conditions, which must hold for all the combinations of the two labor-composite intermediates, $q_{1}, q_{2}$ on the frontier ${ }^{7}$ :

\footnotetext{
${ }^{5}$ In the Foreign country, the production function of each sector are the same, but the factor endowments are exactly symmetric

${ }^{6}$ In particular we need $\Phi=\left(\delta^{\delta}(1-\delta)^{(1-\delta)}\right)^{-1}$

${ }^{7}$ Recall: $q_{1}=l_{1}^{\delta} h_{1}^{1-\delta}, q_{2}=l_{2}^{1-\delta} h_{2}^{\delta}$
} 


$$
\begin{gathered}
r_{L} \bar{L}=\delta v_{1} q_{1}+(1-\delta) v_{2} q_{2} \\
r_{H} \bar{H}=(1-\delta) v_{1} q_{1}+\delta v_{2} q_{2}
\end{gathered}
$$

These two equations express the fact that the total return to factors, unskilled and skilled labor, whose endowment in home country is $\bar{L}$ and $\bar{H}$ respectively, must exhaust completely the total value of the intermediate labor inputs, which is the value added in production in each sector. Substituting 25and26 into 27 and 28 and dividing by $r_{H}$ we get the following two conditions, which define the PPF for $q_{1}$ and $q_{2}$, once $R=r_{L} / r_{H}$ has been eliminated:

$$
\begin{gathered}
R \bar{L}=\delta R^{\delta} q_{1}+(1-\delta) R^{1-\delta} q_{2} \\
\bar{H}=(1-\delta) R^{\delta} q_{1}+\delta R^{1-\delta} q_{2}
\end{gathered}
$$

Spanning different levels of $R$, corresponding to different points on the PPF, we can derive also the share of unskilled labor $\left(l_{1} / \bar{L}\right)$ and of skilled labor $\left(h_{1} / \bar{H}\right)$ used in sector one for each $q_{1}, q_{2}$ combination. The slope of the PPF, defined by the negative of the ratio of the relative "production" costs of the labor composites $\left(-v_{1} / v_{2}\right)$, can be written using 25 and 26 , as $R^{1-2 \delta}$ which is the marginal rate of transformation between labor composite of industry 1 and labor composite of industry 2 .

The representative agent in this type of economy is a household-entrepreneur, who owns a representative share of the skilled and unskilled workers in the economy, and decides how to allocate them between the two sectors in order to maximize the revenues from the sales of the produced goods. A convenient way of representing the problem is to think that the households are entitled to the value added they produce $\left(w_{1} q_{1}^{i}+w_{2} q_{2}^{i}\right)$ and they have a "technology" to transform $q_{1}$ into $q_{2}$ which can be summarized by the function $f\left(q_{1}\right)$, the PPF, implicitly defined by 29 and 30 .

\subsection{Bifurcation Diagram}

The endowments of Skilled and Unskilled Workers is different for the two countries, so that each has a comparative advantage in one sector, in particular the home country is unskilled-Labor abundant and the foreign country is skilled-Labor abundant. We also assume that the relative endowments of unskilled and skilled labor for the two countries are in the range which satisfies the Hecksher-Ohlin conditions for incomplete specialization. In this case, with zero trade cost, we would have incomplete specialization of each country and factor price equalization (FPE). For the simplified assumption of perfect symmetry between the two goods (in the utility function), and between the two 
countries, we may characterize very easily the condition which guarantees incomplete specialization and FPE under free trade. The price of the goods in the two sectors is equal in equilibrium. This implies the same reward to both factors $r_{L}=r_{H}$, (as can be derived from equations 25 and 26) so that the optimal factor ratio in sector 1 will be $l_{1} / h_{1}=\frac{\delta}{1-\delta}$ and in sector 2 will be $l_{2} / h_{2}=\frac{1-\delta}{\delta}$. Therefore, as we are assuming the same amount of skilled and unskilled labor in the world, the condition for being in the FPE cone is that the inequality:

$$
\frac{1-\delta}{\delta}<\left(\frac{\bar{L}}{\bar{H}}\right)_{i}<\frac{\delta}{1-\delta} \quad i=\text { Home, Foreign }
$$

holds for both countries. Under this assumption, the economy under analysis will be subject to two types of forces promoting specialization; the first are the agglomeration economies which are prevailing at intermediate level of transport costs, and work for either sector, once it starts agglomerating; the second are the comparative advantages, which works to concentrate sector one in home and sector two in foreign . Comparative advantages will prevail for high or low transport cost, generating incomplete specialization, while agglomerating economies will prevail in the intermediate range generating full agglomeration, potentially also in the comparatively dis-advantaged sector. The set of relevant values of $q_{1}$ for different values of $T$ (trade costs), is summarized in the so-called "bifurcation diagram" shown in Figure9. In the static models, (see Fujita et al [6]) this diagram used to be the foundation of the stability analysis, now it only provides the description of the "singular" points in the $q_{1}$ space which are to be analyzed to understand the stability. We will use the word "potential" equilibria, rather than equilibria in the description of this diagram, as it is only with the phase-diagram analysis which we could define the stability properties of these points. If, for a value of $T$, more than one value of $q_{1}$ is reported, this means that there are multiple potential equilibria for that value of the parameter. The parameter values (see Appendix) are chosen so that when the home country fully specialized in sector 1 total production in that sector $\left(q_{1}\right)$ is $1^{8}$.

When transport costs are very high (Larger than $T_{1}$ in the figure) there is only one potentially stable equilibrium, with the home country (partially) specialized in sector 1 , so that $q_{1}$ is larger than 0.5 but smaller than 1 . As costs decrease below $T_{1}$ a new potential equilibrium in complete specialization arises, while the partially specialized equilibrium disappears below $\mathrm{T}_{2}$. As transport cost continue to decrease we enter the range in which agglomeration economies are strongest, and for a range between $\mathrm{T}_{3}$ and $\mathrm{T}_{4}$ also the specialization of home in sector 2 is sustainable. Below this point

\footnotetext{
${ }^{8}$ The solid line is for "potentially stable" while the dotted line is for "potentially unstable" equilibria.
} 


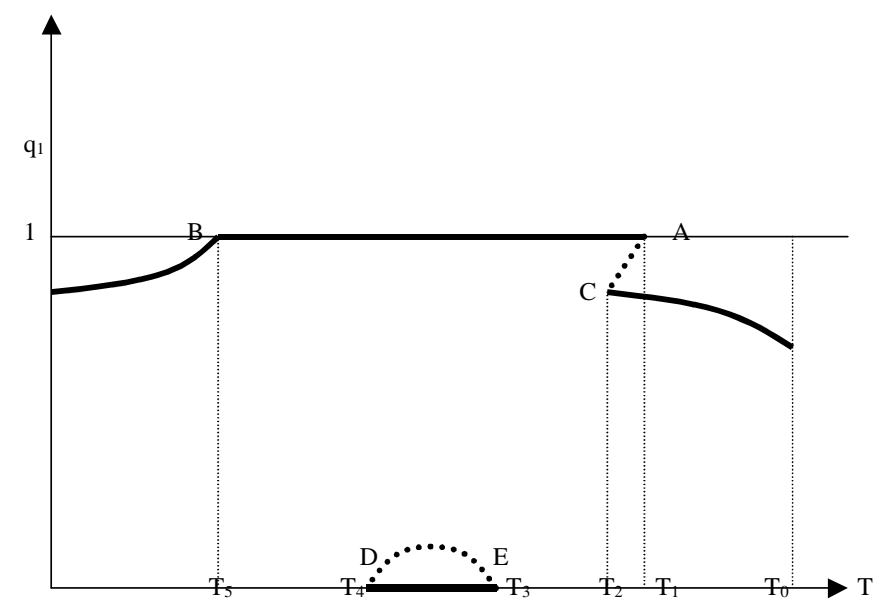

Figure 9: Potential Equilibria in the $q_{1}$ space, for different values of $T$.

the agglomeration economies begin to loose strength, and below $\mathrm{T}_{5}$ only the partially specialized potential equilibrium exists as comparative advantages are what drive the pattern of specialization.

\section{Dynamics and Equilibria with Comparative Ad- vantages}

With the notation defined in the previous section, we can write the instant utility of the household, as sum of the value added in the two sectors, less a term which represents the frictional loss in moving factor across sectors and keeps into account the labor market rigidities which make shifting factors more costly the faster this is done. Therefore the problem of the representative household will be to maximize her utility subject to a constraint of shifting labor between sectors:

$$
\begin{gathered}
\max \left[\int_{t=o}^{\infty} e^{r t}\left(q_{1 i} w_{1}+f\left(q_{1 i}\right) w_{2}-\frac{\gamma m^{2}}{2}\right) d t\right] \\
\text { s.t. } \quad m=\dot{q_{1 i}}
\end{gathered}
$$

The discount rate, equal to the interest, rate is denoted with $r$, and the rate at which production is moved between sectors is $m$, and it involves some frictional costs which are quadratic with a coefficient $\gamma$. The first order conditions for this maximization are: 


$$
\begin{gathered}
\frac{\partial H}{\partial m}=\gamma m-\lambda=0 \\
\lambda=r \lambda-\frac{\partial H}{\partial q_{1 i}}=r \lambda-w_{1}-f^{\prime}\left(q_{1 i}\right) w_{2} \\
\lim _{t \rightarrow \infty} e^{-r t} \lambda=0
\end{gathered}
$$

Now we recall that the first derivative of the function $f$ which transform $q_{1 i}$ into $q_{2 i}$ is the marginal rate of transformation between the two goods which is given by the technological conditions and in equilibrium should be equal to the ratio of prices $\left(w_{2} / w_{1}\right)$. We denote, as developed in the static model, the marginal rate of transformation between the two goods with the notation $\left(-\nu_{1} / \nu_{2}\right)$, as its value is negative. We can then re-arrange the first order conditions, into this system of differential equations, which holds for the aggregate of all agents:

$$
\begin{gathered}
\dot{q}_{1}=\frac{\lambda}{\gamma} \\
\dot{\lambda}=r \lambda-\left(w_{1}-\frac{\nu_{1}}{\nu_{2}} w_{2}\right)
\end{gathered}
$$

This system allows us to analyze the stability property of the different equilibria. For decreasing values of the trade cost, the function $\left(w_{1}-\frac{\nu_{1}}{\nu_{2}} w_{2}\right)$, which depends on $q_{1}$ and will be denoted as $\Psi\left(q_{1}\right)$, will change shape and this is what drives the dynamic change from an equilibrium to another. Here we will analyze only the potential dynamics at some focal points:

When trade cost fall below the level that allows full agglomeration to arise $\left(\mathrm{T}_{2}\right.$ in Figure 9).

When trade costs fall below the level which allows incomplete specialization to rise again ( $T_{5}$ in Figure 9).

\subsection{Dynamics Towards Specialization}

To characterize fully the dynamics of this model in the transitional phase we need to know the shape of the phase diagram before and after the change. This way we could also analyze the effects of a change in expectations, before the actual drop in trade cost takes place. For relatively high trade cost $(T=1.7)$, assuming that sector one is 
intensive in unskilled labor and the home country is abundant in unskilled labor ${ }^{9}$, the function $\Psi\left(q_{1}\right)$ for the home country is reported in Figure $10^{10}$.

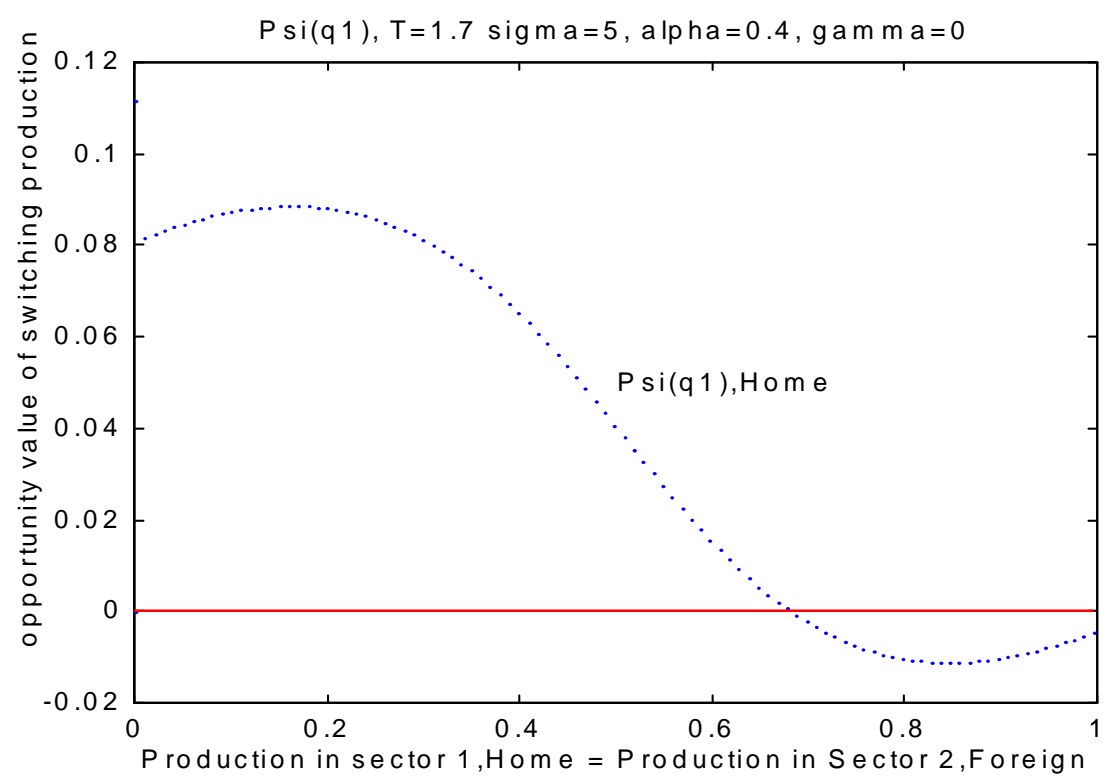

Figure 10: $\Psi\left(q_{1}\right)$ for $\mathrm{T}=1.7$

The only sustainable equilibrium is the one where home is partially specialized in sector one, as in full specialization there are incentives to shift resources towards production in sector 1 . When the trade costs fall below the treshold at which the partially specialized equilibrium disappears, then the function $\Psi\left(q_{1}\right)$ will become as in Figure 11 . Trade costs are, in this case, $\mathrm{T}=1.5$.

The phase diagram, in the case of high trade cost is exactly as in Figure 4, except that point $\mathrm{D}$, rather than being in the middle is placed in $q_{1}>0.5$ as shown in Figure 10. Once trade cost decreases and the function $\Psi\left(q_{1}\right)$ has become as in 11 then the phase diagram is as shown below (Figure12):

As the system is initially in point D, the only solution, satisfying the first order conditions and leading to a stationary asymptotic equilibrium is to jump to point A, as soon as the trade cost drop, and then move gradually, on the trajectory leading to $\mathrm{C}$. In this case the cost of the labor market frictions, in moving from one sector

\footnotetext{
${ }^{9}$ We assume that the relative endowment is in the cone which would ensure incomplete specialization under zero transport costs.

${ }^{10}$ The parameters values of the simulation are reported in appendix
} 


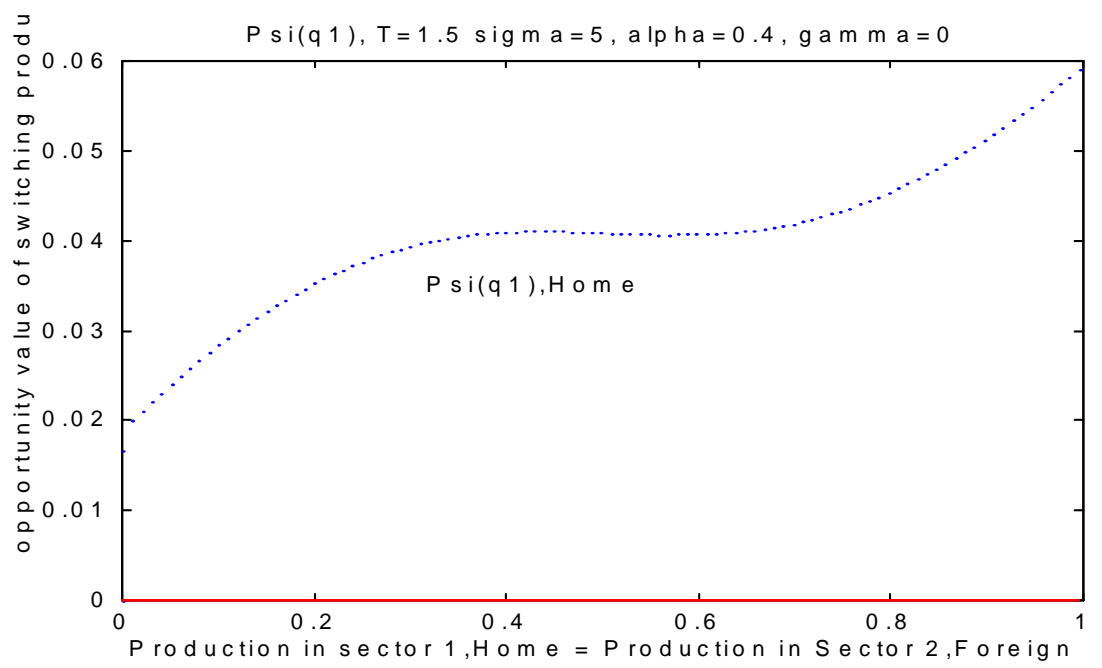

Figure 11: $\Psi\left(q_{1}\right)$ for $\mathrm{T}=1.5$

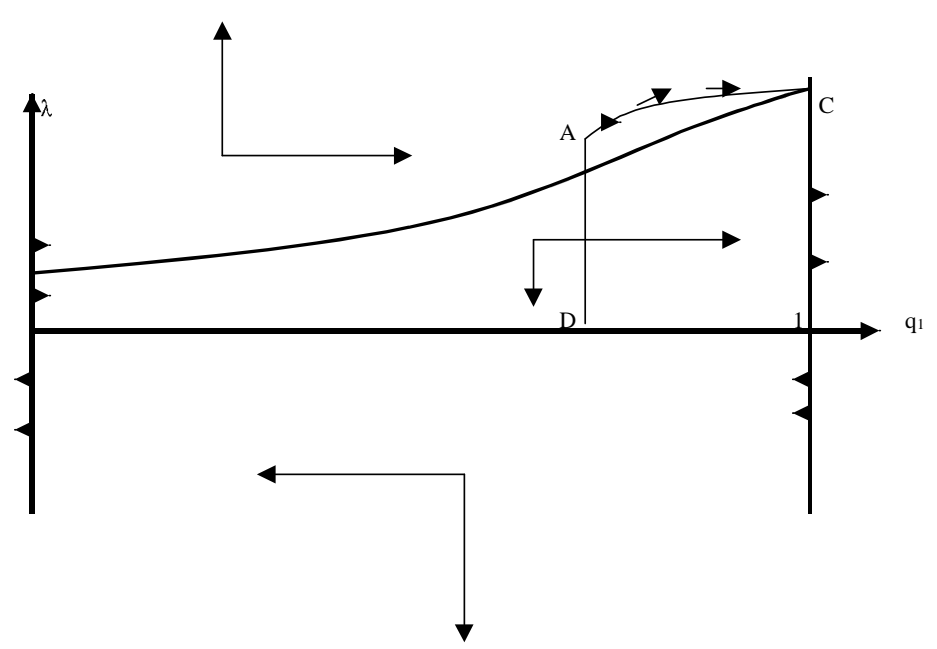

Figure 12: Phase Plan, $\mathrm{T}=1.5$

to the other may affect the speed of the specialization process, but for any value of those frictions full specialization will arise eventually. The "comparative advantage" forces at work in this model are sufficiently strong, when added to the agglomeration economies, to force the system into full specialization even if the frictional cost to move factors between sectors are high. 


\subsection{Unraveling of Complete Specialization}

For an interval of trade costs the agglomeration forces combined with comparative advantages keep the home country fully specialized in sector 1 . Nevertheless, when trade cost becomes lower and agglomeration economies less relevant, the fully specialized equilibrium becomes unsustainable, as the pressure on the factor price, used intensively becomes too high. Therefore there will be another change in the phase diagram which will pass from being basically as in Figure11 to being as in Figure13 below.

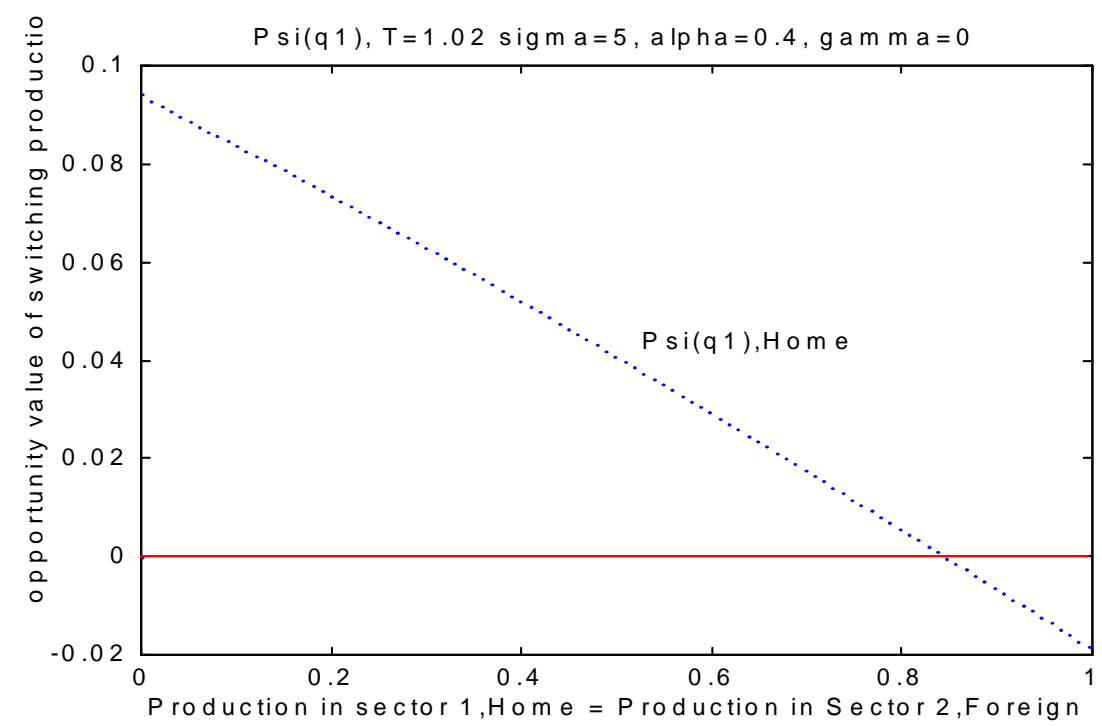

Figure 13: $\Psi\left(q_{1}\right)$ for $T=1.03$

As soon as the trade cost has dropped, the shadow value of moving factors of production to sector one becomes negative, and it is therefore optimal to start moving part of the skilled and unskilled labor away from this sector. The optimal initial speed is given by the shadow value in point $\mathrm{A}$ in Figure14, which is on the saddlepath to the equilibrium D. The optimal and only path leading to the steady state D is therefore the saddlepath of the diagram towards the equilibrium D.

It is possible to study analytically the (local) stability properties of the equilibrium D. As the condition to have the function $\Psi\left(q_{1}\right)$ shaped as in figure 14 is that $d \Psi\left(q_{1}\right) / d q_{1}<0$ at the equilibrium $\mathrm{D}$, then the characteristic roots of the linearized system of differential equations around $\mathrm{D}$, are one positive and one negative, for any value of the labor market rigidities $\gamma$ : 


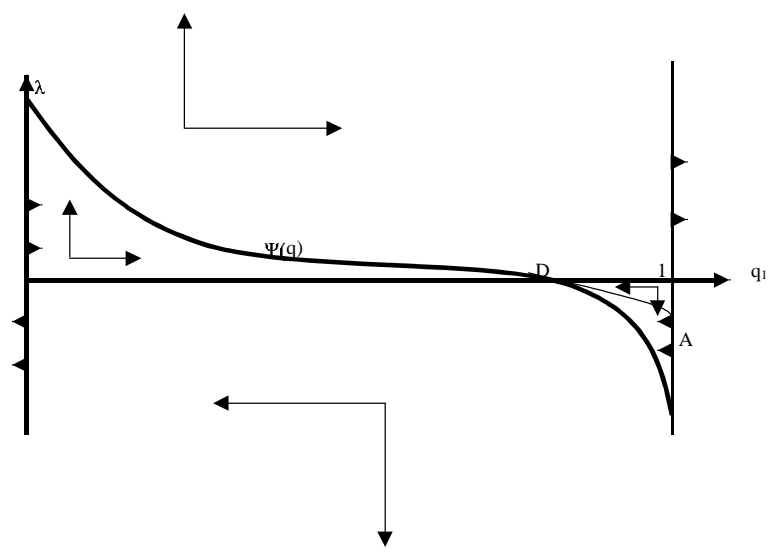

Figure 14: Phase Plan $\mathrm{T}=1.03$

$$
\mu_{1,2}=\frac{r \pm \sqrt{r^{2}-\frac{4}{\gamma} \frac{d \Psi\left(q_{1}\right)}{d q_{1}}}}{2}
$$

This ensures that there is always a unique saddlepath leading to the equilibrium D such as it is shown in Figure 14.

\section{Conclusions}

As different and contrasting forces are at work in the process of globalization and all are affecting the benefits and the costs of international specialization we need a unified frame in which to consider and compare them and their effects. Are comparative advantages stronger than agglomeration economies? Are labor-market frictions a hurdle to specialization? How do these factors interact in a world of increasingly free trade? Even recent and realistically simulated models, such as Forslid et al. [5], while taking into account very carefully the agglomeration economies and the comparative advantages, miss the forward looking behavior and the real dynamics of the model.

The present paper develops a unified model in which forward looking, optimizing households decide how to allocate factors of production between two sectors, in order to maximize their welfare. We are able to show that, lacking comparative advantages, agglomeration economies may not be enough to ensure the emergence of specialization, as globalization proceeds, in the presence of strong labor market rigidities. On the other 
hand, if these rigidities are small, or some comparative advantages, ensured by factor endowments are at work, specialization will increase, at least for intermediate values of trade cost. In both cases, if specialization will arise, the economy will begin to specialize even before the actual drop in trade cost, if there is a reasonable expectation that trade "free-ness" will increase

We are tempted to apply this model to some stylized facts of the western economies. The delay and slow speed at which specialization and industrial agglomeration has been taking place in Europe (only in the 80's according to Amiti[2] and Brulhart and Torstensson[4]), although expected decrease in trade cost have taken place since the 70 's, is probably due to low labor market flexibility which is slowing workers reallocation. Across industrial economies, whose factor endowments are rather similar, so that there are not strong comparative advantage forces, labor market rigidities may generate a prohibitive hurdle for specialization to arise. In this perspective, labor market rigidities have a further cost, besides those usually emphasized; they might slow or, even worse, prevent the process of specialization denying the gains from agglomeration economies. On the other hand, the rapid rise in regional specialization in the US, until the 50's and then its decrease (as documented in Kim [7]), could be the mark of more flexible labor markets and the sign that agglomeration economies have exhausted their effects, leaving the way to a reduction in specialization, generated by the excessive pressure on factor prices in some regions.

The model is a very important step towards the analysis of optimal dynamics during sector and regional booms (specialization) or boosts (de-specialization), and it paves the way for more realistic political analysis of these phenomena, and to the analysis of gain and losses from specialization We have assumed the existence of one representative household which allocate both types of labor but it would be possible and interesting to consider the effect of conflicting interest groups each representing one type of labor. Our contribution defines the tools and some important insights of a potentially rich branch of analysis in dynamic economic geography.

\section{Appendix}

Parameters' value for the simulation in Section 2

$\alpha=0.4, \gamma=0, \beta=0.6, \sigma=5, \bar{L}=1$

Parameters' value for the simulation shown in Section 4 :

$\alpha=0.4, \gamma=0, \delta=0.6, \beta=0.6, \sigma=5 \bar{L}=0.55, \bar{H}=0.45, \tilde{\bar{L}}=0.45, \widetilde{\bar{H}}=0.55$ 


\section{References}

[1] Aigigner K. (1999) "Do industrial Structure converge?" mimeo European Commission DG3 report

[2] Amiti, M (1997).: "Specialization Patterns in Europe," CEP Discussion paper \# 363, London School of Economics, 1997.

[3] Baldwin R.(1999) "The Core-Periphery model with Forward-Looking Expectations" NBER W.P. \# 6921, Feb 1999

[4] Brulhart, M. and J. Torstensson (1996): "Regional Integration, scale economies and industry location in the European economy," CEPR Discussion paper \# 1435, 1996.

[5] Forslid R., J. Haaland and K.H.M. Knarvik (1999) :" A U-shaped Europe? a simulation study of industrial location" CEPR Discussion Paper, September 1999

[6] Fujita M., P. Krugman and A.J. Venables (1999) "The Spatial Economy" MПT Press Cambridge, London 1999

[7] Kim S. (1995): "Expansion of Markets and the Geographic Distribution of Economic Activities: The Trend in U.S. Regional Manufacturing Structure, 18601987" Quarterly Journal of Economics 1995

[8] Krugman P. (1991a) "Increasing Returns and Economic Geography" Journal of Political Economy 99: 483-499

[9] Krugman P.(1991b) "Geography and Trade" MIT Press, Cambridge New England 1991

[10] Krugman P. and A.J. Venables (1995) "Globalization and the inequality of Nations" Quarterly Journal of Economics 110(4): 857-880

[11] Wolfmayr-Schnitzaer Y.(1999) "Globalization, Integration and Specialization of Countries: a survey on Literature " WIFO working Paper 1999. 